\title{
Hacia la definición conceptual del constructo Calidad de la Educación Superior en el contexto de la Universidad Nacional Agraria
}

Araica Zepeda, Ricardo; Vargas Hernández, Marcia Mercedes

Hacia la definición conceptual del constructo Calidad de la Educación Superior en el contexto de la Universidad Nacional Agraria

Revista Educación, vol. 44, núm. 2, 2020

Universidad de Costa Rica, Costa Rica

Disponible en: http://www.redalyc.org/articulo.oa?id=44062184039

DOI: https://doi.org/10.15517/revedu.v44i2.40522

Esta obra está bajo una Licencia Creative Commons Atribución-NoComercial-SinDerivar 3.0 Internacional. 


\title{
Hacia la definición conceptual del constructo Calidad de la Educación Superior en el contexto de la Universidad Nacional Agraria
}

\author{
Towards a Conceptual definition for the Construct, Higher Quality of Education within the Context of the \\ National Agricultural University, Nicaragua
}

Ricardo Araica Zepeda

Universidad Nacional Agraria (UNA), Nicaragua

ricardo.araica@ci.una.edu.ni

http://orcid.org/0000-0001-6516-3896

Marcia Mercedes Vargas Hernández

Universidad Nacional de Ingeniería (UNI), Nicaragua

marcia.vargas@fiq.uni.edu.ni

(iD) http://orcid.org/0000-0001-7774-0806

\author{
DOI: https://doi.org/10.15517/revedu.v44i2.40522 \\ Redalyc: http://www.redalyc.org/articulo.oa?id=44062184039
}

Recepción: 10 Marzo 2020

Aprobación: 29 Junio 2020

\section{Resumen:}

El propósito principal de este artículo es definir el constructo Calidad de la Educación Superior en el contexto de la Universidad Nacional Agraria (UNA); mediante el análisis comparativo de las dimensiones de consistencia interna y externa, a partir de un planteamiento preliminar elaborado con base en la revisión de literatura de autores de América Latina y El Caribe. Esta valoración tomó en consideración la perspectiva del enfoque sistémico y la teoría de los constructos personales. Con base en un enfoque cualitativo se fortaleció la conceptualización del constructo, mediante indagación empírica en la cual participaron ocho expertos: cinco nacionales y tres internacionales entrevistados personalmente, excepto dos casos vía correo electrónico. Para el análisis de la información obtenida de las entrevistas semiestructuradas se utilizó el Método Comparativo Constante, apoyado de la herramienta informática MAXQDA. El análisis sistémico y el enfoque holístico elaborado posibilitó la valoración del planteamiento inicial con los resultados de la investigación de campo, identificándose rasgos emergentes, entre los que se destacaron: la calidad como construcción social; la transversalidad de la calidad al quehacer institucional; su naturaleza dinámica y relativa; y, por último, la satisfacción de expectativas, vista desde el impacto, pertinencia y contribución a la transformación social. La definición de este constructo es un marco referencial del Sistema Gestión y de Aseguramiento de la Calidad, de la mejora continua y del fortalecimiento de la cultura de la calidad en la UNA; que por su complejidad y dinamismo debe ser valorado de forma permanente. Palabras Clave: Calidad, Consistencia interna, Consistencia externa, Educación Superior, Enfoque sistémico.

\section{Abstract:}

This study defines the construct for the Quality of Higher Education within the context of the National Agricultural University (Universidad Nacional Agraria (UNA)) in Nicaragua, using a comparative analysis of internal and external consistency dimensions, which, in turn is based on a preliminary approach from a literature review by Latin American and Caribbean authors. The methodology included a systemic approach and personal construct theory based on a qualitative approach. The construct was conceptualized and strengthened through empirical research involving eight experts. The five national and three international experts were interviewed in person with the exception of two who were interviewed via email. Data analysis was based on semistructured interviews, use of the Constant Comparative Method and the MAXQDA software program. Field research enabled a systemic analysis and holistic approach which identified the following emerging features: the social construction of quality, the cross-cutting nature involving the concept of quality at an institutional level, its dynamic and relative nature, and, finally, fulfillment of expectations regarding impact, relevance and contribution to social transformation. The definition of this construct serves as a reference framework for the Quality Management and Assurance System which provides continuous improvement and strengthening of the UNA quality culture which, given its complex and dynamic nature, requires continuous assessment.

KEYWORDS: Quality, Internal Consistency, External Consistency, Higher Education, Systemic Approach. 


\section{INTRODUCCIÓN}

Uno de los referentes internacionales para indagar sobre el significado y la cobertura de la Calidad de la Educación Superior es el Tratado Bolonia del año 1999, dándose cambios significativos en la formación universitaria en un espacio europeo que fuera competitivo y atractivo a nivel mundial. Algunos de los cambios fueron: adaptaciones curriculares, tecnológicas y la adopción de un sistema de titulaciones universitario comparable en toda Europa que coadyuvó a la movilidad de estudiantes, docentes, mientras promovía el aprendizaje continuo y la calidad de la Educación (Lago, Gamboa y Montes, 2014). Igualmente, es fundamental valorar la Calidad de la Educación Superior desde sus actores claves: estudiantes y docentes; así como también desde los discursos universitarios y desde la sociedad en general. En ese sentido, es importante determinar el modo de ver la educación y sobre todo la visión relativa a la importancia de la formación profesional en el desarrollo integral de la sociedad.

Uno de los determinantes principales de Calidad de la Educación Superior para el estudiantado es la función docente, esto significa valorar, un profesor de calidad. Otro determinante que sobresale es la gestión universitaria, vista desde la garantía de la sostenibilidad, bienestar y servicios universitarios. Desde el profesorado se arguye también como relevante la función docente, vista de una forma más amplia, tanto la calidad de la enseñanza como la calidad del aprendizaje; además, se distinguen otros factores, tales como: la investigación, la extensión universitaria, el estado de la infraestructura, bibliotecas, entre otros; y desde las Universidades, la mirada es aún más amplia abarca desde la filosofía institucional, marco legal, políticas, los procesos universitarios de docencia, investigación, extensión y gestión; y sus niveles de respuestas a las demandas de la sociedad (Lago, et al., 2014).

En Nicaragua, la Constitución Política en su artículo 125 otorga a las Universidades autonomía, sus derechos, deberes y la responsabilidad del Estado de financiar la Educación Superior pública. Particularmente, la Ley $N^{\circ} 89$ (1990), Ley de Autonomía de las Instituciones de Educación Superior, les otorga autonomía académica, financiera, orgánica y administrativa; así mismo, establece el Consejo Nacional de Universidades (CNU). A finales del año 2019 el Consejo Nacional de Evaluación y Acreditación (CNEA) dio a conocer el Modelo de Calidad de la Educación Superior de Nicaragua (CNEA, 2019a), y el glosario de Educación Superior de Nicaragua (CNEA, 2019b), en que plantea la definición de calidad de la educación superior, con base en un análisis documental.

En ese sentido, es perentorio tomar en consideración el posicionamiento de los actores y expertos en gestión universitaria nacionales e internacionales para su apropiación e internalización. Por tanto, el propósito principal de este artículo es definir el constructo Calidad de la Educación Superior en el contexto de la Universidad Nacional Agraria; desde una perspectiva holística, conjugada con el análisis de los rasgos intrínsecos y consustanciales de este constructo en el contexto de nicaragüense y regional. La estructura de contenido de este artículo contiene los siguientes apartados: Resumen, Introducción, Antecedentes prácticos o Estado de la cuestión, Referentes conceptuales o marco teórico, Procedimientos metodológicos, Análisis y discusión de resultados, Conclusiones y Referencias.

\section{ANTECEDENTES PRÁCTICOS O ESTAdo DE LA CUESTión}

\section{Contexto nacional}

En Nicaragua no se registran investigaciones empíricas sobre la conceptualización de calidad en la educación superior. Solamente se cuenta con leyes nacionales que describen de manera general sobre la calidad de la educación superior. En ese sentido, la Asamblea Nacional de la República de Nicaragua [AN] en la Ley N. 582 (2006), Ley General de Educación, define en el capítulo III, artículo N. 6, inciso e, el concepto de Calidad 
de la Educación, en la que se destaca la pertinencia del diseño curricular y la relevancia del aprendizaje para la vida de los educandos. Asimismo, la AN en la Ley No 89 (1990), Ley de Autonomía de las Instituciones de Educación Superior, establece que el Consejo Nacional de Universidades (CNU) es la instancia de coordinación y asesoría de las Universidades y Centros de Educación Técnica Superior; encargada de elaborar y coordinar la política nacional de la Educación Superior del país; velar por que cumplan con la formación de profesionales de acuerdo con los fines y objetivos de las Instituciones de Educación Superior nicaragüense definidas por esta ley; autorizar la creación de nuevas instituciones; dictaminar sobre la apertura o cierre de carreras y proponer la política de distribución de los fondos asignados por el Estado a las Instituciones miembros.

Además, se cuenta con la Ley $N^{\circ} 704$ (2011), Ley creadora del Sistema Nacional para el Aseguramiento de la Calidad de la Educación y Reguladora del Consejo Nacional de Evaluación y Acreditación (CNEA), la cual mandata en su Título Tercero, Capítulo I, Arto. 9, lo siguiente:

Cada institución universitaria establecerá dentro su organización un sistema de aseguramiento de la calidad, mediante el cual se gestionará los procesos encaminados a asegurar la mejora continua de la calidad. Su forma de organización y funcionamiento será determinada por la propia institución en el ejercicio de su autonomía (AN, p. 5967).

En el año 2013, 55 universidades de Nicaragua, en cumplimiento a la Ley $\mathrm{N}^{\circ} 704$ iniciaron un primer proceso obligatorio de Autoevaluación Institucional con fines de mejoras, culminando en el año 2018 con la implementación de su plan de mejoras. En esta materia la Universidad Nacional Agraria (UNA) declara en el Modelo y Proyecto Educativo (2011) un acápite denominado Sistema de Aseguramiento de la Calidad, en el que se plantea de manera general lo que se concibe como calidad y algunos aspectos de este.

\section{Contexto Internacional: Calidad de la Educación Superior}

El concepto de calidad ha evolucionada en cada época histórica, abarcando todos los ámbitos de la vida social, transitando desde la producción al sector servicios; y en particular a la educación, lo cual es corroborado por Águila (2005):

Hasta comienzos de la década del 60 del siglo pasado, existía una visión tradicional y estática de la calidad de la educación universitaria, se presuponía la calidad de la enseñanza y el aprendizaje como constitutivos del sistema, se basaba en la tradición de la institución, en la exclusividad de profesores, alumnos y en los recursos materiales (p. 2).

Además, arguye que, cambia de contenido en cada época, no es estable y duradero porque es un concepto primordialmente histórico. Actualmente, existen numerosos planteamientos destacándose el de la Red Iberoamericana de Aseguramiento de la Calidad de la Educación Superior (2004) que define la calidad como:

Grado en que un conjunto de rasgos diferenciadores inherentes a la educación superior cumple con una necesidad o expectativa establecida, se refiere al funcionamiento ejemplar de una IES. También, la define como propiedad de una institución o programa que cumple los estándares previamente establecidos por una Agencia u organismo de Acreditación. Para medirse adecuadamente suele implicar la evaluación de la docencia, el aprendizaje, la gestión y los resultados obtenidos. Cada parte debe ser medida por su calidad y el conjunto supone la calidad global (p.15).

En ese mismo sentido, Vlăsceanu, Grünberg, y Pârlea (2004) plantean:

Calidad en la educación superior como un concepto multidimensional de múltiples niveles, dinámico, que se relaciona con los elementos contextuales de un modelo educacional, con la misión y fines institucionales, y con estándares específicos dentro de un sistema, institución, programa o disciplina determinados. La calidad, por tanto, puede adquirir significados diferentes dependiendo de (i) la comprensión de los diversos intereses de distintos grupos comprometidos o actores en la educación superior; (ii) sus referencias: Insumos, procesos, productos, misiones, objetivos, etc.; (iii) los atributos o características del mundo académico que se considera necesario evaluar; y (iv) el período histórico en el desarrollo de la educación superior (p. 315). 
Desde otra perspectiva, García (2016) afirma que: "la educación superior se ha convertido en un factor clave del progreso económico, cultural y social de cualquier país” (p. 84).

A pesar de ser abundante la información documental sobre Calidad de la Educación Superior, especialmente los dedicados al tema de Calidad; no existe un concepto unívoco, que responda a la naturaleza y contexto económico, político, jurídico, social y cultural de los países de América Latina y El Caribe. Lo cual, es válido para el caso de Nicaragua; y, por ende, para la Universidad Nacional Agraria, con base en la naturaleza de su contexto.

\section{REFERENTES CONCEPTUALES O MARCO TEÓRICO}

Debido a la naturaleza multidimensional y complejidad de las universidades, se requiere abordar la Calidad de la Educación Superior desde un enfoque holístico, por sus múltiples escenarios y contextos en que se desarrolla su quehacer. Con base en lo anterior, la perspectiva teórica central utilizada fue la Teoría General de Sistemas (TGS) que se considera una metateoría y de formas complementarias se utilizaron las Teorías del Desarrollo Organizacional; Análisis sistémico cuya base teórica y metodológica son el enfoque sistémico y la teoría general de los sistemas; y la Teoría de la Psicología de los Constructos Personales.

Según Cathalifaud y Osorio (1998):

Si bien el campo de aplicaciones de la TGS no reconoce limitaciones, al usarla en fenómenos humanos, sociales y culturales se advierte que sus raíces están en el área de los sistemas naturales (organismos) y en el de los sistemas artificiales (máquinas) (p. 3).

En ese mismo sentido Chiavenato (2006) plantea:

Las organizaciones no están aisladas ni son autosuficientes, sino que forman parte de un universo más amplio. Son sistemas que actúan dentro de otros sistemas y están insertas en un medio constituido por otras organizaciones, en una relación de interdependencia que les permite sobrevivir y competir en un mundo complejo (p. 415).

Lo antes expuesto, es plenamente pertinente al caso de las Universidades que son sistemas sociales, complejos y abiertos; que, además, en el marco de su autonomía se organizan estructuralmente como subsistemas, para atender las funciones sustantivas de: docencia, investigación, extensión y gestión, en cumplimiento con su misión al servicio de la sociedad.

\section{Primera aproximación conceptual del constructo de Calidad de la Educación SUPERIOR}

Desde la epistemología crítica, el posicionamiento teórico sobre Calidad de la Educación Superior se concibe como un constructo multidimensional, polisémico, relativo y dinámico, que se construye socialmente, partiendo de la filosofía institucional (misión, visión, principios y valores), planes y capacidades para cumplir determinados estándares nacionales e internacionales; y satisfacer los requerimientos de formación profesional que demanda el desarrollo económico, político y social en el entorno en que interactúa. Posteriormente, este constructo fue fortalecido con la investigación no experimental, lo que implicó la valoración de expertos nacionales e internacionales entrevistados; tomando en cuenta el contexto latinoamericano, regional y nacional, por eso, es una primera aproximación a la definición conceptual de este constructo el cual es un continuum, perfectible que implica un análisis riguroso y confiable, como parte de la dialéctica de la construcción del conocimiento y la práctica histórica-ontológica del investigador. 


\section{Procedimientos metodológicos}

El enfoque metodológico utilizado fue el sistémico y la Teoría Psicológica de los Constructos Personales de George A. Kelly (1905-1966), que para sentar sus bases conceptuales partió de un postulado fundamental, a saber: "Los procesos de una persona se canalizan psicológicamente por las formas en que anticipa los acontecimientos" (Botella y Feixas, 1998, p. 39). La etapa de indagación se desarrolló desde un enfoque cualitativo, mediante la Técnica de la entrevista semiestructurada: "la cual se basa en una guía de asuntos o preguntas en la cual el entrevistador tiene la libertad de introducir preguntas adicionales para precisar y obtener más información sobre los temas en cuestión” (Hernández, Fernández y Baptista, 2006, p. 597).

Los criterios de selección de los expertos entrevistados fueron: a) Formación académica posgraduada; b) Experiencia profesional de más de 20 años en la educación superior; c) Experiencia laboral en gestión universitaria y en organismos de aseguramiento de la calidad de la educación superior; d) Perteneciente a países de Centroamérica y América Latina; y e) Disposición de brindar la entrevista semiestructurada. La indagación empírica contó con el aporte de ocho expertos: cinco de Nicaragua -de los cuales uno es de la universidad objeto de estudio- y tres internacionales de Guatemala, Costa Rica y Chile; a seis de ellos se le aplicó la entrevista personalmente, y a las otras dos, vía correo electrónico.

La valoración de los datos cualitativos se caracterizó por una serie de procedimientos sistematizados y optimizados mediante el uso de un programa informático, manteniendo la calidad, la pluralidad de tipos de análisis y las características esenciales del enfoque cualitativo. Este enfoque fue respaldado por un conjunto de criterios de evaluación para garantizar el rigor científico y metodológico, entre los cuales se destacan: reflexividad, coherencia, transparencia, credibilidad, perspectiva holística, sistematicidad metodológica y conciencia de complejidad (Gehrig et al., 2014). Para lograr la consistencia de los datos se emplearon procedimientos específicos tales como la triangulación de fuentes de información y entre autores, utilizando el Método Comparativo Constante (MCC) de Glaser y Strauss (1967), y luego modificado por Lincoln y Guba (1985). Según Ramallo y Andrés, (2008), este método:

Implica la codificación y el análisis simultáneo de datos a fin de desarrollar conceptos. A través de la comparación constante se logra mayor precisión de los conceptos, el reconocimiento de sus propiedades, el conocimiento de sus interrelaciones y finalmente la integración en una teoría congruente (p. 4).

Los principales tópicos abordados durante las entrevistas fueron: las principales características de la educación superior, enfoque de calidad y el concepto de calidad en la educación superior desde su experiencia profesional. Posteriormente, fueron procesadas con la herramienta informática MAXQDA (Analysis of qualitative data, por sus siglas en inglés).

\section{ANÁLISIS Y DISCUSIÓN DE LOS RESULTADOS}

A continuación, se presentan los resultados del procesamiento de las entrevistas aplicadas a ocho expertos, cinco nacionales y tres internacionales, enfatizando sobre el constructo Calidad de la Educación Superior, para lo cual se decidió agrupar los rasgos en dos dimensiones: consistencia interna y consistencia externa, basado en los planteamientos de Lemaitre y Zenteno (2012); Pedraja y Rodríguez (2013).

\section{Dimensión 1: Análisis de la consistencia interna}

Para objeto de este estudio la consistencia interna del constructo Calidad de la Educación Superior, implica: desarrollar un quehacer institucional consistente con los propósitos, objetivos y promesas elaboradas autónomamente por la propia entidad. En ese sentido, se estableció que existen cuatro rasgos intrínsecos 
y consustanciales al constructo Calidad de la Educación Superior, tales como: filosofía institucional; multidimensional; transversalidad y relatividad, los cuales se detallan a continuación:

La Filosofía Institucional, fue el rasgo más señalado por los expertos entrevistados, arguyendo que Calidad en la Educación Superior es: "Actuar conforme a su misión, visión y objetivos y consecuente con sus valores" (Entrevista 1, comunicación personal, 13 de octubre 2017). "Un programa universitario o una institución de educación superior es de calidad sí cumple con sus objetivos” (Entrevista 2, comunicación personal, 10 de octubre 2017).

De este modo, la calidad en la educación superior puede entenderse por una parte como la [que] cumple con sus objetivos, es decir, de la promesa que la institución hace a la sociedad y a sus estudiantes; por otra, como la verificación de la pertinencia de esos propósitos, de la promesa institucional (Entrevista 4, comunicación personal, 11 de mayo 2017).

Para la conceptualización y/o definición del constructo de calidad, considero que se debe tomar en cuenta el marco filosófico, las políticas de la universidad como punto de partida, en el entendido que los elementos del marco filosófico marcan el camino a seguir en todo el quehacer de la universidad y las políticas marcan el camino para la definición de estrategias que se operativizan en los planes estratégicos (Entrevista 5, comunicación personal, 15 de mayo 2017).

"Al cumplimiento de la misión y objetivos de la institución si yo no cumplo con lo que me propongo no estoy haciendo algo de calidad y digamos hacerlo mejor podría hacerlo con calidad" (Entrevista 6, comunicación personal, 18 de octubre 2017).

Lo anteriormente expresado es compartido por Castaño y García (2012) sobre que:

La calidad es un concepto relativo asociado al proyecto institucional, a sus objetivos, finalidades y resultados, a su eficiencia y costo, a su aptitud para cumplir su compromiso social o bien para satisfacer las necesidades de sus usuarios, destinatarios o clientes (p. 221).

En este mismo sentido, la Organización de las Naciones Unidas para la Educación, la Ciencia y la Cultura [UNESCO] (1998) y Tapia (2013), afirman que la calidad es la adecuación del Ser y Quehacer de la Educación Superior a su Deber ser. De acuerdo con la experiencia internacional el atractivo de esta definición radica en su flexibilidad inherente, la que permite a las instituciones medir su calidad en términos de su capacidad para cumplir sus respectivas misiones y objetivos (Yamada y Castro, 2013).

Un segundo rasgo destacado fue la Multidimensionalidad, cuyas aseveraciones fueron las siguientes: "Tienen que lograr que sus resultados que son los graduados alcancen las competencias esperadas por el mundo del trabajo, desarrollen la capacidad para aprender, transformarse y responder a los cambios y demandas de sector" (Entrevista 2, comunicación personal, 10 de octubre 2017).

Entonces, todos los grandes pensadores dijeron entonces si es multidimensional este tema de la calidad. Entonces, ¿cuáles son los docentes, las infraestructuras, los planes de estudios, la gestión, la investigación, la extensión? pero no fue suficiente, porque dentro de cada uno de estos había otro montón de cosas (Entrevista 7, comunicación personal, 11 de octubre 2017).

"Esta debe ser entendida de manera multidimensional pues ha de asegurar una formación integral de los estudiantes, debiendo para ello de asegurar el poseer estudiantes sobresalientes, académicos destacados" (Entrevista 8, comunicación personal, 26 de septiembre 2017).

Según la Organización de las Naciones Unidas para la Educación, la Ciencia y la Cultura [UNESCO] (1998) en la Declaración Mundial sobre Educación Superior en el Siglo XXI: Visión y Acción, en el artículo 11 se expresa claramente sobre la calidad de la enseñanza superior lo siguiente:

La calidad de la enseñanza superior es un concepto pluridimensional que debería comprender todas sus funciones y actividades: enseñanza y programas académicos, investigación y becas, personal, estudiantes, edificios, instalaciones, equipamiento y servicios a la comunidad y al mundo universitario. Una autoevaluación interna y un examen externo realizados con transparencia por expertos independientes, en lo posible especializados en lo internacional, son esenciales para la mejora de la calidad (pp. 10-11). 
De igual manera, "las declaraciones de Dakar, Tokio y Beirut (1997) presentan conceptos de la calidad que también están en consonancia con las definiciones establecidas en el Documento de política para el cambio y el desarrollo en la educación superior de la UNESCO” (López, 2012, p. 629). Adicionalmente, Yamada y Castro (2013); Royero (2002); Águila (2005); Sanatuario (2007); Aguerrondo (1993); concuerdan que la calidad es multidimensional en que se incluyen todas las funciones universitarias, en concordancia con lo planteado por la UNESCO (1998).

Un tercer rasgo señalado destacado fue la Transversalidad del constructo Calidad de Educación Superior, planteada de la siguiente manera: "Esta definición incluye aspectos tales como el peso relativo de las funciones institucionales, las áreas del conocimiento en las que espera desarrollar su quehacer, el tipo de estudiantes que decide admitir" (Entrevista 4, comunicación personal, 11 de mayo 2017).

Y como un eje transversal, de manera que sea parte permanente de la vida institucional, por ende, en la definición del constructo de calidad. Estas indican la ruta, los procesos y los resultados a obtener que den cuenta a lo interno y externo de la universidad a través de las funciones sustantivas que de manera articulada ejecuta (Entrevista 5, comunicación personal, 15 de mayo 2017).

El Consejo Nacional de Acreditación de Colombia afirma que la calidad de la educación es un atributo de servicio público de la educación en general, y en particular, al modo como este servicio se presta, según el tipo de institución de que se trate (CNA, 2013, p.11).

Además, se alude que el esfuerzo continuo de las instituciones por cumplir de forma responsable con las exigencias propias de cada una de las funciones universitarias.

En esa misma dirección, Orellana (2015) argumenta que la garantía de calidad institucional es naturalmente transversal a las disciplinas, y asume un enfoque propiamente organizacional en todo su quehacer.

Un cuarto y último rasgo identificado por los expertos entrevistados fue la Relatividad, expresando lo siguiente:

Bueno, si usted le pregunta: ¿qué es un programa de calidad?, en todo este proceso probablemente van a persistir respuestas distintas, dependiendo de a quién le está preguntando y no existen respuestas mejores que otras, es decir, son respuestas distintas, son igualmente legítimas (Entrevista 2, comunicación personal, 10 de octubre 2017).

Al final de cuenta la calidad, es bueno resaltar dos cosas muy importantes. Uno que es relativa y otra que es comparativa; es decir, vos podés determinar si algo es de calidad en la medida que podas comparar con algo que ya está preestablecido, lo podés comparar entonces la única forma de reconocer si algo es de calidad o no lo es (Entrevista 7, comunicación personal, 11 de octubre 2017).

Lo cual es consistente con lo planteado por González y Espinoza (2008) en torno a la relatividad por varias razones: a) por quién usa el término y las circunstancias por las cuales lo invoca; b) es de naturaleza similar a la verdad y la belleza; y por eso, constituye un ideal difícil de comprometer; y c) conlleva los valores del usuario y, en ese sentido, es subjetivo. Castaño y García (2012) plantean que:

Si bien existen multiplicidad de acepciones de lo que a calidad se refiere. Esta no es un término absoluto, sino que por el contrario es un término relativo y dependiente de todos los factores que constituyen el micro y macroentorno de los centros de enseñanza (p. 221).

Con base en lo anterior, Sanatuario (2007) refiere que para el Instituto Nacional para la Evaluación de la Educación (INEE): "el concepto de calidad es relativo, porque el juicio depende del punto de referencia adoptado" (p. 23).

\section{Dimensión 2: Análisis de la consistencia externa}

La consistencia externa del constructo implica desarrollar un quehacer institucional consistente con las exigencias del medio externo y los estándares exigidos por la comunidad científica o profesional. Asimismo, 
se determinó que existen seis rasgos intrínsecos y consustanciales al constructo Calidad de la Educación Superior, tales como.Estándares, criterios y procedimientos; satisfacción de expectativas; construcción social; contextualización; dinamismo; comparabilidad; y aptitud para el uso; los cuales se detallan a continuación. En general, todos los expertos coincidieron en señalar el rasgo de Estándares, criterios y procedimientos, como intrínseco y consustancial, mediante los siguientes argumentos: "Yo creo que ahí entran los sistemas nacionales de evaluación y acreditación que deben de poner estándares mínimos, que realmente se reflejen en la vida del país" (Entrevista 1, comunicación personal, 13 de octubre 2017). "Entonces, una institución con un programa de calidad tiene que transformarse y responder a los cambios y demandas de su entorno y hacerlo con pertinencia y transparencia basado en principios y valores, es un esfuerzo de mejoramiento continuo" (Entrevista 2, comunicación personal, 10 de octubre 2017).

Hay que tener referentes para saber a qué se refiere la experiencia que se vive en la universidad: El currículo, los docentes, los estudiantes, la infraestructura, los resultados y unos criterios de valoración: la pertinencia, la equidad, la suficiencia, la eficacia y la eficiencia, entre otros (Entrevista 3, comunicación personal, 09 de mayo 2017).

"Y la capacidad de respuesta a necesidades y demandas diferentes; al mismo tiempo, exige a los organismos de aseguramiento de la calidad adecuar los indicadores relativos a recursos, procesos y resultados" (Entrevista 4, comunicación personal, 11 de mayo 2017). "Así mismo, incorporar el criterio de calidad como criterio de evaluación, como principio" (Entrevista 5, comunicación personal, 15 de mayo 2017). "Bueno, a mí me parece que la calidad tiene que verse como un conjunto de propiedades, de estándares, de indicadores mínimos que todos deberíamos de cumplir en la educación superior" (Entrevista 6, comunicación personal, 18 de octubre 2017). "Bueno, cuando hablamos de los estándares del CNEA [Consejo Nacional de Evaluación y Acreditación] lo que pensamos hacer [son] estándares que sean contextualizados, entonces hablamos de estándares previamente consensuados, socializado con las instituciones" (Entrevista 7 , comunicación personal, 11 de octubre 2017).

En el mundo los sistemas de aseguramiento de calidad se vienen consolidando por la necesidad de contar con criterios validados de comparación entre instituciones y entre programas de estudio, en respuesta a los cuestionamientos a la legitimidad y calidad de la oferta educativa que se generan con el crecimiento sustantivo de la cobertura de educación superior (Entrevista 8, comunicación personal, 26 de septiembre 2017).

De igual modo, Royero (2002); González y Espinoza (2008); basándose en la clasificación de Harvey y Green (1993), señalan este rasgo como la calidad como excepción, la cual en su tercera variante la vincula al cumplimiento de estándares mínimos, lo cual posibilita tener distintos conjuntos de estándares para diferentes tipos de instituciones. Castaño y García (2012) reafirman:

Que el concepto de calidad en un inicio se asociaba a los procesos industriales, de ahí la definición tradicional del concepto de calidad que da Green (1994) en el que se le asocia a la idea de ofrecer un producto o servicio distintivo o especial, que implica estándares elevados en la elaboración o entrega de ese producto o servicio y que ofrece un estatus especial a quien lo produce u ofrece; pero este concepto no es muy adecuado cuando se trata de calidad en la educación (p. 221).

En ese mismo sentido, Yamada y Castro (2013) y Águila (2005); plantean que el concepto de calidad como excelencia, está basado en la definición tradicional, equivalente a poseer estudiantes sobresalientes, académicos destacados, y aseguramientos del primer nivel.

Un segundo rasgo del constructo destacado fue la Satisfacción de expectativas, con base en los siguientes planteamientos:

Eso digamos es para la institución si uno lo ve a nivel de país yo creo que la calidad tiene que estar medida por el grado de conformidad que hay entre lo que la universidad hace y lo que el país está esperando y demandando de sus instituciones (Entrevista 1, comunicación personal, 13 de octubre 2017).

Logra que sus graduados alcancen las competencias esperadas por sus pares profesionales y académicos y otros sectores de la sociedad, muestra capacidad para aprender, transformarse, responder a los cambios y demandas de su entorno, con 
pertinencia, transparencia en un proceso de mejoramiento continuo fundamentado en principios y valores (Entrevista 2, comunicación personal, 10 de octubre 2017).

"La consistencia externa o la capacidad para identificar las necesidades o demandas que emergen del medio externo pertinente. Asimismo, a definir con claridad los resultados que ofrece a la sociedad” (Entrevista 4, comunicación personal, 11 de mayo 2017).

\footnotetext{
Luego, tendría que ver si la pertinencia yo la pondría entre la duda, pero si definitivamente lo que yo quiero ver es como se integra el tema de la investigación, de extensión dentro del proceso de enseñanza-aprendizaje, para efectos de poder darles respuesta a la sociedad (Entrevista 7, comunicación personal, 11 de octubre 2017).

Finalmente, considero que la definición de calidad debe de tener el cuidado de que al considerar la pertinencia de los procesos que intervienen en la formación del profesional no se corra el peligro de que la calidad se tome solamente sobre la base de los requerimientos puramente técnicos y no los aspectos culturales y de valores en los graduados universitarios (Entrevista 8, comunicación personal, 26 de septiembre 2017).
}

En concordancia con lo antes planteado, Águila (2005) reafirma "que la pertinencia y la calidad, junto a la internacionalización, representan para la UNESCO, los tres aspectos claves que determinan la posición estratégica de la educación universitaria" (p. 2). Para INEE (2006), "la calidad educativa comprende varias dimensiones: la pertinencia y la relevancia que expresan la coherencia entre la enseñanza y las necesidades de los alumnos y la sociedad" (p. 9).

Con referencia a lo anterior, Águila (2005) advierte que el concepto de calidad como respuesta a los requerimientos del medio, provocaría entre otras cosas el abandono de la misión universitaria como difusora de la cultura y la degradación de la formación de los profesionales. "Pero al mismo tiempo limitaría al profesional egresado para realizar la necesaria movilidad e intercambio con otras regiones del país y del mundo" (Águila, 2005, p. 4).

Un tercer rasgo del constructo Calidad de la Educación Superior, que fue mencionado por cuatro de los ocho expertos entrevistados fue la Construcción social, con base en las siguientes explicaciones: "El concepto de calidad es un concepto que se construye de manera social y se construye socialmente, para recoger las miradas de los diferentes sectores de la sociedad" (Entrevista 2, comunicación personal, 10 de octubre 2017).

Sería apropiado utilizar técnicas propias del meta plan en donde la gente con libertad puede dar sus aportes y después de manera grupal pueden ir construyendo categorías, que le permitirán tipificar las diferentes maneras de comprender esa calidad. Esa será la base para la construcción del constructo de calidad en el contexto en el que realiza el estudio (Entrevista 3, comunicación personal, 09 de mayo 2017).

"La calidad es también una construcción social. Entonces, al ser la calidad una construcción social también cuando definimos esos profesores entonces es una construcción social también particular en un contexto particular" (Entrevista 6, comunicación personal, 18 de octubre 2017).

El planteamiento anterior evidencia que en Educación Superior el asunto de la calidad debe ser analizado y discutido en la comunidad académica considerando educación pública y privada, si se piensa que es posible poder determinar un concepto de calidad única para Nicaragua (Entrevista 8, comunicación personal, 26 de septiembre 2017).

Análogamente, González y Espinoza (2008) reafirman que: "el concepto de calidad es una construcción social, que varía según los intereses de los grupos de dentro” (p. 252) y fuera de la institución. También, Royero (2002); Aguerrondo (1993); coinciden en afirmar que la calidad es una categoría social e históricamente determinada, su concepción obedece a manifestaciones concretas de los distintos modos de formación económica social en el cual se incluye.

Un cuarto rasgo del constructo de Calidad de la Educación Superior, que fue mencionado por tres de los ocho expertos entrevistados fue la Contextualización, con base en los siguientes argumentos: "El concepto de calidad no puede entenderse separado del contexto en el que se desempeña la institución o la carrera cuya calidad se quiere evaluar" (Entrevista 4, comunicación personal, 11 de mayo 2017). "El contexto es 
clave también, puesto que contribuye a ubicar y redireccionar las acciones de la universidad" (Entrevista 5 , comunicación personal, 15 de mayo 2017).

"Y se debe de interpretar como el resultado de cambios internos y externos que afectan los distintos procesos institucionales, por lo que es importante que su definición está determinada por aquella que más convenga a la realidad que vive" (Entrevista 8, comunicación personal, 26 de septiembre 2017).

$\mathrm{Al}$ respecto, Salcebo (1998), plantea:

El estudio del concepto de calidad no debe sólo conformarse con el análisis de los atributos o rasgos que lo caracterizan, sino que es necesario, además, ubicarlo en el contexto sociocultural e histórico del cual es parte la institución o programa objeto de evaluación (p. 76).

En ese mismo sentido, Garduño (1999) reafirma que "la calidad de la educación está histórica y culturalmente especificada, y se construye en cada espacio" (p. 97). Asimismo, Águila (2005) afirma que "el concepto de calidad en la educación universitaria cambia de contenido en cada época, es decir, no es estable y duradero porque se trata de un concepto primordialmente histórico" (p. 2).

Un quinto rasgo del constructo Calidad de la Educación Superior, que fue mencionado por dos de los ocho expertos entrevistados fue el Dinamismo, con base en las siguientes explicaciones:

Si usted le pregunta a un profesor, por ejemplo, sobre todo aquellos profesores que son digamos acuciosos y muy vinculados
a la investigación y al trabajo académico y se lo toman muy en serio estos profesores probablemente van a juzgar la calidad
del programa dependiendo en qué medida los contenidos del programa están cerca de lo que podríamos llamar la frontera
del conocimiento de su disciplina, él está pensando en las fronteras de la ciencia, en los avances de la ciencia (Entrevista 2 ,
comunicación personal, 10 de octubre 2017).

"Es importante tener claro que el concepto de calidad de la educación universitaria es cambiante, no es estable y duradero porque es un concepto dinámico, tanto en el ámbito mundial como local” (Entrevista 8 , comunicación personal, 26 de septiembre 2017).

Sanatuario (2007) reafirma que es dinámico, porque la calidad nunca se alcanza del todo y siempre pueden proponerse metas superiores. La calidad no es un estado sino una tendencia; la auto exigencia permanente y razonable de superación, surge del interior del sistema a mejorar [el cual es retroalimentado por las demandas sociales externas]. Este mismo autor, comparte el planteamiento de la ANUIES (2006), sobre la calidad como: "un concepto dinámico y producto del acuerdo entre los actores claves, de los entornos y proyectos institucionales; de los valores y visiones del futuro que orientan las actividades" (p. 82).

Un sexto rasgo del constructo Calidad de la Educación Superior, que fue mencionado por dos de los ocho expertos entrevistados fue la Comparabilidad, con base en las siguientes argumentaciones:

Si usted le pregunta a un estudiante probablemente él va a estar más pensando, un programa de calidad es aquel que me da las competencias que me va a permitir conseguir un buen trabajo, tener éxito en mi profesión y desempeñarme exitosamente en la práctica y conseguir un buen trabajo eso es lo que el que estudiante está pensando, si el programa me ofrece eso, es de calidad para mí (Entrevista 2, comunicación personal, 10 de octubre 2017).

Y otra que es comparativa; es decir, vos podés determinar si algo es de calidad en la medida que podas comparar con algo que ya está preestablecido, lo podés comparar entonces [como] la única forma de reconocer si algo es de calidad o no lo es (Entrevista 7, comunicación personal, 11 de octubre 2017).

Coincidentemente, Garduño (1999) profundiza que:

Cuando se expresa la calidad de un objeto como un juicio, inmediatamente se establecen, cuando menos, tres supuestos: 1) una referencia a ciertas características del objeto; 2) un conocimiento real o inferido de la persona que lo expresa sobre la participación de las características y sus relaciones; y 3) una comparación del objeto con algún tipo de referente o con otros objetos similares (p. 98).

Por tanto, en rigor sólo se puede establecer que una institución es mejor que otra cuando son homólogas en sus fines, concordantes en su misión y en un contexto similar. 
Un séptimo rasgo del constructo de Calidad de la Educación Superior, que fue mencionado por uno de los ocho expertos entrevistados fue la Aptitud para el uso, con base en las siguientes argumentaciones:

\begin{abstract}
Bueno, no creo que exista una definición exacta de lo que es calidad en la educación superior, yo creo que la calidad tendría que estar referida a lo que ya varios autores han dicho al cumplimiento para hacer bien las cosas, y que debe de ser de estricto cumplimiento para la formación de nuestros graduados, que están definidos ya sea en la misión o están definidos externa o internamente con la institución que le permiten que ella pueda realmente cumplir con su función social, que es la función de formar a los graduados y aportarle al desarrollo social al país, entonces así lo miraría yo y el concepto de calidad vista en esa perspectiva (Entrevista 6, comunicación personal, 18 de octubre 2017).
\end{abstract}

En ese mismo sentido, Royero (2002) afirma que: "la calidad como valor agregado supone que las instituciones de educación superior deben ante el ente financista, generalmente es el Estado, rendir cuentas mediante evidencias que representen logros socialmente válidos" (p. 2). Igualmente, Yamada y Castro (2013) concluyen:

Por ello, en adelante, al referirnos a calidad educativa estaremos hablando del proceso a través del cual se imparten un conjunto de conocimientos y herramientas específicas, y se muestra cómo combinarlos con las habilidades básicas que ya tiene el estudiante, para producir una tarea valorada en el mercado de trabajo (p. 28).

No obstante, existen diversos autores que coinciden con una de las acepciones de la tipología propuesta por Harvey y Green (1993), referida a la calidad como transformación del estudiante, entre los cuales se pueden mencionar: Yamada y Castro (2013); Royero (2002); Aguerrondo (1993); Garbanzo (2007); enfatizan que la educación no es un servicio para el cliente, sino un proceso continuo de transformación de las y los participantes, mediante cambios y mejoras de las actividades cognitivas de las y los estudiantes en el logro de los objetivos y políticas de la educación, en aras del desarrollo social y la dignidad humana. Por tanto, se plantea que los rasgos inherentes al concepto Calidad de la Educación Superior, antes mencionados del contexto interno y externo son congruentes a las condiciones contextuales, naturaleza y características de las Universidades públicas nicaragüenses; y en particular, de la Universidad Nacional Agraria (UNA, 2011), declarado en la filosofía institucional. Con base en lo anterior se efectuó una valoración desde la epistemología critica, el análisis sistémico y el enfoque holistico, estructurándose el constructo en cuestión de la siguiente manera:

Calidad de la Educación Superior: Es un constructo polisémico, constituido por un conjunto de rasgos intrínsecos y consustanciales, tales como: multidimensional, transversal, relativo, dinámico, comparable, aptitud para el uso y transformación; que se construye socialmente, partiendo de la filosofía institucional (misión, visión, principios y valores), planes, funciones sustantivas y capacidades institucionales, para la mejora continua, el cumplimiento de estándares, el logro de aprendizajes significativos en la formación profesional y humanística, para la búsqueda de la excelencia; en función de satisfacer las expectativas de los actores sociales internos y externos (estudiantes, docentes, administrativos, empleadores y sociedad en general) comprometidos con la pertinencia, el desarrollo sostenible y la transformación tecnológica, productiva, política y social en la que interactúa, en cada momento histórico.

\title{
Conclusiones
}

La definición de un constructo surge de la necesidad humana de generalizar y armonizar referencias; son formulaciones mentales de la realidad y de lo que puede percibir de ella, las cuales se aplican, designan o clasifican a los diversos objetos, hechos, procesos y situaciones vividas; que constituyen experiencias, únicas e irrepetibles. En consecuencia, son considerados delimitaciones fundamentales para investigaciones educativas de sistemas educativos complejos.

Se han expuesto diversos planteamientos sobre calidad; y en particular, Calidad en la Educación Superior, quedando plenamente evidenciado que existe una diversidad de rasgos intrínsecos y consustanciales a este 
concepto polisémico. Asimismo, el uso de las dimensiones consistencia interna y externa, posibilitó una valoración holística. El análisis sistémico desarrollado posibilitó la valoración del posicionamiento teórico inicial con los resultados de la investigación empírica, lográndose identificarlos rasgos más destacados que en orden de prelación fueron los referidos a: los estándares, requisitos o criterios de calidad, satisfacción de las expectativas, multidimensionalidad y la Filosofia Institucional siendo coincidentes con los hallazgos empíricos reportados en la literatura consultada. Asimismo, fueron identificados rasgos emergentes, entre los que sobresalieron: la calidad como construcción social; la transversalidad de la calidad al quehacer institucional. su naturaleza dinámica y relativa (estas dependen del contexto y de los actores sociales); y, por último, la satisfacción de expectativas vista desde el impacto, pertinencia y contribución a la transformación social.

El constructo definido es consistente con la Agenda 2030, a partir de su objetivo 4: Garantizar una educación inclusiva y equitativa de calidad y promover oportunidades de aprendizaje permanente para todos, en la que se articulan los objetivos 5, 10,13,15, 15 y 16, para garantizar la formación integral de los profesionales [consistencia interna]; y posteriormente, los objetivos 2, 3, 6, 7, 8, 9, 11 y 12, que reafirman la pertinencia de su quehacer [consistencia externa] (Bidegain, 2017); la Declaración de la CRES-1998, en lo relativo a que la que la calidad de la educación superior es pluridimensional, debe ser pertinente, eficiente y comprometida con el desarrollo sostenible (UNESCO, 1998); y la Tercera Conferencia Regional CRES-2018, que reafirma el tema de la calidad, en torno a la diversidad y pertinencia social (UNESCO, 2018). En este sentido, estos resultados son coherentes con la filosofía, Modelo y Proyecto Educativo de la UNA. En consecuencia, este constructo es plausible y valido para la realidad contextual de esta institución. Es importante, denotar que este constructo constituye un marco referencial del Sistema Gestión y de Aseguramiento de la Calidad, de la mejora continua y del fortalecimiento de la cultura de la calidad en la universidad. Sin embargo, se sugiere que este constructo por su complejidad y dinamismo debe ser valorado de forma permanente, con base en las tendencias del desarrollo de la educación superior y las demandas cambiantes y crecientes con las necesidades del entorno, que coadyuve al desarrollo de una gestión universitaria efectiva.

\section{REFERENCIAS}

Aguerrondo, I. (1993). La calidad de la educación: ejes para su definición y evaluación. Revista interamericana de desarrollo educativo, 37(116), 561-578. Recuperado de https://bit.ly/2vGmobv

Águila, V. (2005). El concepto calidad en la educación universitaria: clave para el logro de la competitividad institucional. Revista Iberoamericana De Educación, 36(12), 1-7. Recuperado de https://rieoei.org/RIE/article /view/2886

Asamblea Nacional de la República de Nicaragua [AN] (1990). Ley N 89. Ley de Autonomía de las Instituciones de Educación Superior. La Gaceta, Diario Oficial N 77, del 20 de abril de 1990, pp. 825-833, Nicaragua. Recuperado de https://www.lagaceta.gob.ni/1990/04/077/

Asamblea Nacional de la República de Nicaragua [AN] (2006). Ley N 582. Ley general de educación. La Gaceta, Diario Oficial N 150, del 3 de agosto de 2006, pp. 6405-6516, Nicaragua. Recuperado de https://www.lagace ta.gob.ni/2006/08/150/

Asamblea Nacional de la República de Nicaragua [AN] (2011). Ley N 704. Ley creadora del sistema nacional para el aseguramiento de la calidad de la educación y reguladora del Consejo Nacional de Evaluación y Acreditación. La Gaceta, Diario Oficial No. 172, del 12 de septiembre de 2011, pp. 5695-5975, Nicaragua. Recuperado de h ttps://www.lagaceta.gob.ni/2011/09/172/

Asociación Nacional de Universidades e Instituciones de Educación Superior [ANUIES]. (2006). Consolidación y avance de la educación superior en México: Elementos de diagnóstico y propuesta. México: ANUIES. Recuperado de http://www.humanindex.unam.mx/humanindex/consultas/detalle_libros.php?id=9389\&rfc=MERJ520903

Bidegain, N. (2017). La Agenda 2030 y la Agenda Regional de Género: sinergias para la igualdad en América Latina y el Caribe. Recuperado de https://bit.ly/36ig6Nk 
Botella, L., y Feixas, G. (1998). Teoría de los constructos personales: Aplicaciones a la práctica psicológica. Barcelona: Laertes. Recuperado de https://bit.ly/39p3hBA

Castaño, G., y García, L. (2012). Una revisión teórica de la calidad de la educación superior en el contexto colombiano. Educación y Educadores, 15(2). Recuperado de http://www.scielo.org.co/pdf/eded/v15n2/v15n2a05.pdf

Cathalifaud, M., y Osorio, F. (1998). Introducción a los conceptos básicos de la teoría general de sistemas. Cinta de Moebio, (3), 40-49. Recuperado de https://www.redalyc.org/pdf/101/10100306.pdf

Chiavenato, I. (2006). Introducción a la Teoría General de la Administración. Séptima edición. Bogotá: McGraw Hill Interamericana. Recuperado de https://esmirnasite.files.wordpress.com/2017/07/i-admon-chiavenato.pdf

Consejo Nacional de Acreditación [CNA] (2013). Acreditación de programas de pregrado en Colombia. Recuperado de https://www.cna.gov.co/1741/article-186377.html

Consejo Nacional de Evaluación y Acreditación [CNEA] (2019a). Modelo de Calidad de la Educación Superior de Nicaragua. Recuperado de

Consejo Nacional de Evaluación y Acreditación [CNEA] (2019b). Glosario de Educación Superior de Nicaragua. Recuperado de

Garbanzo, M. (2007). Calidad y equidad de la educación superior pública. Aspectos por considerar en su interpretación. Revista Educación, 31(2), 11-27. Recuperado de https://doi.org/10.15517/revedu.v31i2.1241

García, E. (2016). Concepto de excelencia en enseñanza superior universitaria. Educación médica, 17(3), 83-87 Recuperado de https://doi.org/10.1016/j.edumed.2016.06.003

Garduño, L. (1999). Hacia un modelo de evaluación de la calidad de instituciones de educación superior. Revista iberoamericana de educación, (21), 93-103.

Gehrig, R.; Palacios, J.; Blesa, B.; Cobo, F.; García, M.; Muñoz, P.; y Rodes, J. (2014). Guía de criterios básicos de calidad en la investigación cualitativa. España, UCAM. Recuperado de https://bit.ly/2IkXYag

Glaser, B., y Strauss, A. (1967). The Discovery of Grounded Theory: Strategiesfor Qualitative Research. New York. Aldine Publishing Company. Recuperado de https://bit.ly/2VIg4ea

González, L. y Espinoza, O. (2008). Calidad de la educación superior: concepto y modelos 2008-09. Calidad en la Educación, (28), 247-276, doi, https://doi.org/10.31619/caledu.n28.210

Green, D. (1994). What is Quality in Higher Education? Society for Research into Higher Education \& Open University Press (London). Recuperado de https://files.eric.ed.gov/fulltext/ED415723.pdf

Harvey, L., y Green, D. (1993). Defining Quality. Assessment y Evaluation in Higher Education, 18(1), 9-34, doi, htt ps://doi.org/10.1080/0260293930180102

Hernández, R., Fernández, C., y Baptista, P. (2006). Metodología de la Investigación (4a edición). Ciudad de México, México: McGraw Hill. Recuperado de https://bit.ly/2w48ujw

Instituto Nacional para la Evaluación de la Educación [INEE] (2006). La calidad de la educación básica ayer, hoy y mañana. Informe anual sobre la calidad de la educación básica en México 2006. México: INEE. Recuperado de $\mathrm{h}$ ttps://www.inee.edu.mx/wp-content/uploads/2018/12/P1B205.pdf

Lago, D., Gamboa, A., y Montes, A. (2014). Calidad de la educación superior: Un análisis de sus principales determinantes. Saber, ciencia y libertad, 9(1), 157-170. Recuperado de https://bit.ly/2VLPW2i

Lemaitre, M., y Zenteno, M. (2012). Aseguramiento de la calidad en Iberoamérica, educación superior, Informe 2012. Chile, Centro Interuniversitario de Desarrollo (CINDA), Universia. Recuperado de https://bit.ly/3ccvKMM

Lincoln, Y., y Guba, E. (1985). Naturalistic Inquiry. Newbury Park: Calif.: Sage Publications Inc. London, New Delhi. Recuperado de https://bit.ly/2TnjOAj

López, F. (2012). La segunda conferencia mundial de educación superior (UNESCO, 2009) y la visión del concepto de acreditación en las conferencias de UNESCO (1998-2009). Avaliação: Revista da Avaliação da Educação Superior (Campinas), 17(3), 619-636. Recuperado de http://www.scielo.br/pdf/aval/v17n3/a04v17n3.pdf

Organización de las Naciones Unidas para la Educación, la Ciencia y la Cultura [UNESCO] (1998). Declaración mundial sobre la educación superior en el siglo XXI: visión y acción. Revista de la Educación Superior, 27(107). Recuperado de https://bit.ly/32ReB79 
Organización de las Naciones Unidas para la Educación, la Ciencia y la Cultura [UNESCO] (2018). Informe General CRES 2018. Congreso llevado a cabo en III Conferencia Regional de Educación Superior. Recuperado de http s://www.iesalc.unesco.org/2018/12/13/informe-general-de-la-cres-2018/

Orellana, V. (2015). Calidad de la educación superior. Elementospara una interpretación sociológica. (Tesis de maestría). Universidad de Chile, Chile. Recuperado de https://bit.ly/2TkJcVZ

Pedraja, L., y Rodríguez, E. (2013). El desafío de la calidad en las universidades. Idesia (Arica), 31(4). Recuperado de https://doi.org/10.4067/S0718-34292013000400001

Ramallo, M., y Andrés, R. (2008). Lo cualitativo, un modelo para la comprensión de los métodos de investigación. Documento de Trabajo $N^{\circ}$ 216. Argentina: Universidad de Belgrano. Recuperado de http://repositorio.ub.edu .ar/handle/123456789/4782

Red Iberoamericana de Aseguramiento de la Calidad de la Educación Superior (2004). Glosario internacional Riaces de evaluación de la calidad y acreditación. Madrid: RIACES. Recuperado de http://www.riaces.org/v1/index.p $\mathrm{hp} /$ servicios/glosario-riaces

Royero, J. (2002). Contexto mundial sobre la evaluación en las instituciones de educación superior. Revista Iberoamericana de Educación edición digital, España, sección lectores. 1-13. Recuperado de https://rieoei.org/his torico/deloslectores/334royero.pdf

Salcebo, H. (1998). Indicadores de gestión para las universidades venezolanas: un proyecto de alcance nacional. Agenda Académica, 6(1), 63-91. Recuperado de https://bit.ly/32UzxKf

Sanatuario, A. (2007). Dimensiones de la calidad en educación superior. REencuentro. Análisis de Problemas Universitarios, (50), 21-27. Recuperado de https://www.redalyc.org/pdf/340/34005004.pdf

Tapia, V. (2013). La gestión de la calidad de la educación universitaria: desafíos y posibilidades. Cultura. Ciencia y Tecnología, ASDOPEN-UNMSM/, (3), 21-29. Recuperado de https://bit.ly/2TmwF5E

Universidad Nacional Agraria [UNA] (2011). Modelo y Proyecto Educativo. Managua, Nicaragua: Universidad Nacional Agraria. Recuperado de https://bit.ly/2Xm2dt8

Vlăsceanu, L., Grünberg, L., y Pârlea, D. (2004). Quality assurance and accreditation: A glossary of basic terms and definitions. Bucharest: Unesco-Cepes. Recuperado de https://bit.ly/2IaAJ2R

Yamada, G., y Castro, J. (2013). Calidad y acreditación de la educación superior: retos urgentes para el Perú. Universidad del Pacífico. Recuperado de https://bit.ly/2PO3QNw

\section{BY-NC-ND}

